\title{
THE RESPIRATION OF THE ISOLATED OUTERSEGMENTS OF RODS
}

\author{
ISAO HANAWA, EIICHI KIMURA AND YUJI HOSOYA* \\ Department of Physiology, Osaka City University Medical School
}

Recently, Ottoson and Svaetichin $(14,15)$ and Svaetichin (18) succeeded in recording electroretinogram with microelectrode inserted into the receptor layer. They concluded that a vertebrate electroretinogram is generated by the photoreceptor cells only and the a-wave of the electroretinogram is generated by the cone and the $b$-wave by the rod. Svaetichin also reported that the fish retina has never revealed any response of rods after their outersegments are removed.

Sjöstrand $(16,17)$ analyzed outer-and innersegments of the rods of guinea pig and perch eyes by means of a high resolution electron microscope using ultrathin sections. He reported that outersegments of rods are composed of a pile of $140 \AA$ thick double membrane discs which are connected together in a series by means of short processes of stalks. The main part of the innersegment consists of long slender mitochondria which are densely aggregated. Viewing this from a morphological organization of the rod, he proposed a hypothesis regarding the function of visual cells that the outersegment receives the light energy which causes excitation that reaches down from the outersegment to the innersegment through the connecting fibril. It is a well established fact that all the rhodopsin to be found is confined to the outersegments of rods where photoreaction is initiated. But the metabolism of the outersegments of rods had been unknown untill Hubbard (8) studied the respiration of the isolated rod outersegment of the frog retina. In investigating the metabolism of the rod cell one problem must be solved, i.e., an applicable method of separation and collection of the outersegments of rods in the pure state. Recently, Kimura (10) reported a new method of separating the outersegments of rods from retinal tissues. This method enables us to study the metabolism of the outersegment of the rod.

The present paper deals with the problems: 1) whether the outersegment of rod can keep respiration in Ringer solution without glucose, 2) whether the respiration of the outersegment is influenced by illumination, 3) whether this respiration is influenced by some metabolic inhibitors.

\section{METHODS}

Experimental animals employed were toads (Bufo vulgaris formosus). They were kept in a dark room overnight and the all operations were carried out

Received for publication August 24, 1955.

* 塙 功 木村英一 細谷雄二 
under dim red light or dim white light, below one lux. The outersegments of rods were isolated by Kimura's new sugar floatation method (10) at low temperature, below $10^{\circ} \mathrm{C}$. The outersegments collected at the boundary between sugar solutions (specific gravities 1.15 and 1.12 respectively) was pipetted off (fig. 1 and fig. 2). In order to remove sugar from the outersegment, 10 volumes of isotonic $\mathrm{NaCl}$ solution were added in a sugar suspension of outersegments, and then centrifuged at 8,000 r.p.m. for 5 min. The outersegments were precipitated at the bottom in a centrifuge tube. The supernatant fluid was discarded, then outersegments were suspended with $5 \mathrm{ml}$. of Ringer solution without glucose, $p_{\mathrm{H}} 7.4$.

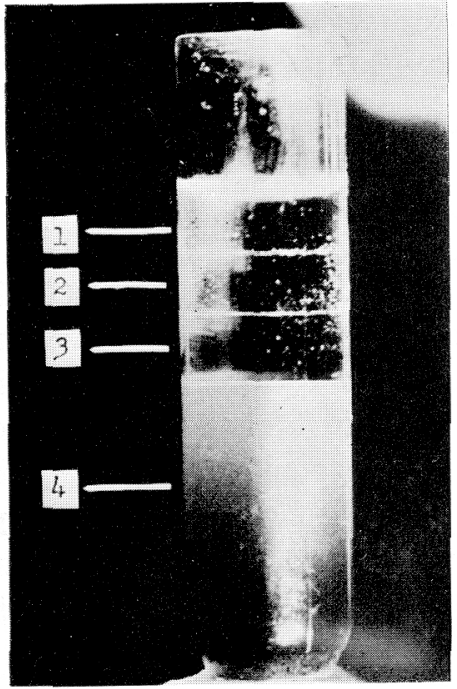

FIG. 1. Four liquid layers in the centrifuge tube.

1. Distilled water.

2. sp. gr. 1.12

3. sp. gr. 1.15 Sugar solutions

4. sp. gr. 1.20

(suspension of crushed retinae.)

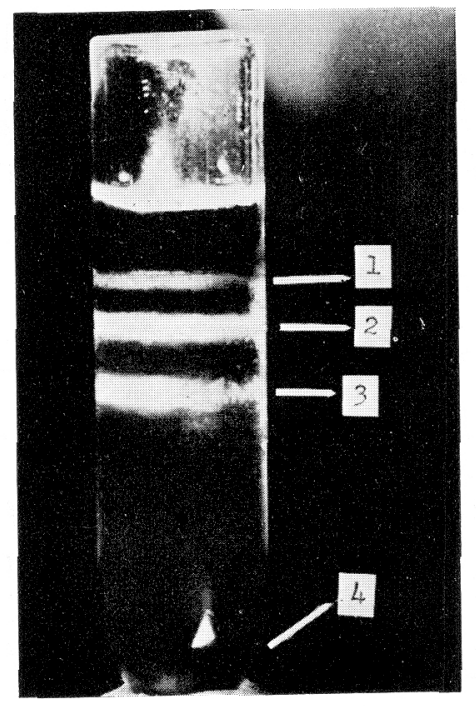

FIG. 2. Separated retinal tissues in the centrifuge tube.

1. Stratum of very fine fragments.

2. Stratum of outersegments of rods.

3. Stratum of innersegments of rods.

4. Pigment epithelium, nuclear and ganglionic layers.

All experiments were carried out at $26^{\circ} \mathrm{C}$. by the Warburg apparatus using Ringer solution without glucose as a suspending medium. In each experiment three Warburg flasks were used. One of these flasks was a thermobarometer. Each $2 \mathrm{ml}$. of outersegments suspension was placed in the other two flasks. To the center wall of each flask, $0.2 \mathrm{ml}$. of $10 \% \mathrm{KOH}$ was added for $\mathrm{CO}_{2}$ absorption. To the side arm, $0.5 \mathrm{ml}$. of Ringer solution without glucose or of some metabolic inhibitors was added. All flasks were equilibrated for 10 minutes in a dark room and readings were taken over a 150 - or 180-minute period.

In each experiment, separation and collection of outersegments was completed within 90 -minute. 
After the measuring of the oxygen consumption, outersegments used in each experiment were centrifuged and dry weights were determined after the sample was allowed to stand for 120 -minutes at $110^{\circ} \mathrm{C}$.

\section{RESULTS}

1) The respiration of outersegments in the dark and under 1 lux white light

When outersegments are separated under dim red light with the darkest possible condition, their oxygen consumption rates are shown in fig. 3 . The outersegment exhibits a remarkable respiring capacity in the dark and the $\mathrm{QO}_{2}$ value based on exygen uptake during the initial 60-minute interval is -28.6. When the same sample is exposed to 1 lux white light during the measurement, its oxygen consumption rate is slightly increased in the early stage, then gradually it shows more decrease than in the non-light treatment. It decreases $5 \%$ during a 180 -minute period. It is very interesting that respiration of outersegments is slightly depressed in the early stage. This depression is remarkable

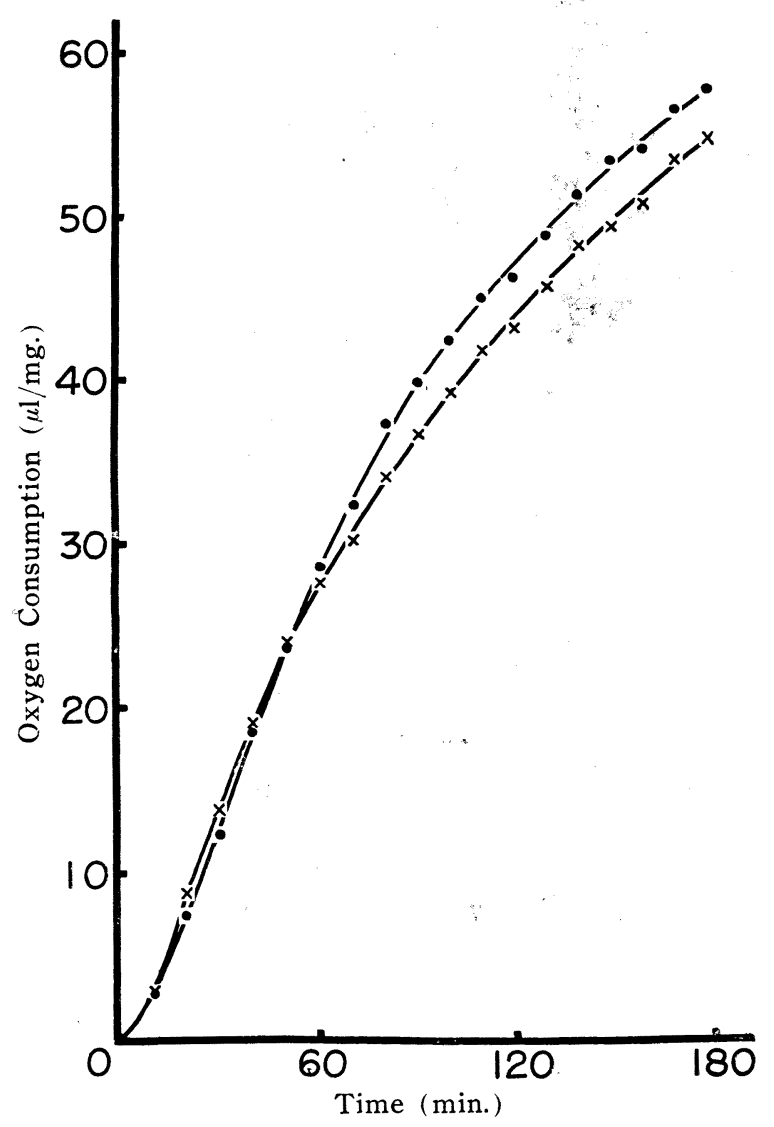
when the procedure of separation is carried out unber a brighter red lamp than the above described conditions. Therefore, dark adapted outersegments collected in an absolutely dark room may not reveal any depression in the early stage, but we can not prove it because such procedure is impossible in absolute darkness.

FIG. 3. Oxygen consumption of outersegments in the dark condition with exposure to 1 lux white light. Experiment carried out in Warburg manometers at $26^{\circ} \mathrm{C}$. All vessels contained 2.5 $\mathrm{ml}$. of suspension of outersegments in Ringer solution without glucose, $p \mathrm{H} 7.4$.

$\bullet-\bullet$, oxygen consumption in the dark.

$x-x$, oxygen consumption when exposed to 1 lux white light. 


\section{2) Effects of 25 or 550 lux white light illumination}

When outersegments are separated in dim white light (below 1 lux), the oxygen consumption rates are revealed as shown in fig. 4 and fig. 5 . In the dark condition during the period of measurement, each oxygen uptake in both cases is depressed remarkably during the first 50-60 minute period, followed by an abrupt increase. These initial depressions may be due to a metabolic change in the outersegment under the illumination while the toads's eyes are being treated. When the same samples are exposed to 25 or 550 lux white light during manometric measurement, each oxygen uptake in both cases is strongly inhibited during a 180-minute period. The inhibition comes up to 75 or $79 \%$ on the oxygen uptake under 25 or 550 lux white light illumination. When the outersegments are placed in the dark after the initial illumination for $90 \mathrm{~min}$., the inhibition of oxygen uptake ceases, resulting in a sudden increase in oxygen consumption rate (fig. 5). On the contrary, no noticeable change is found in oxygen consumption rate, when the outersegments are illuminated by 550 lux white light, after they are placed in the dark during the first 90 minute period.

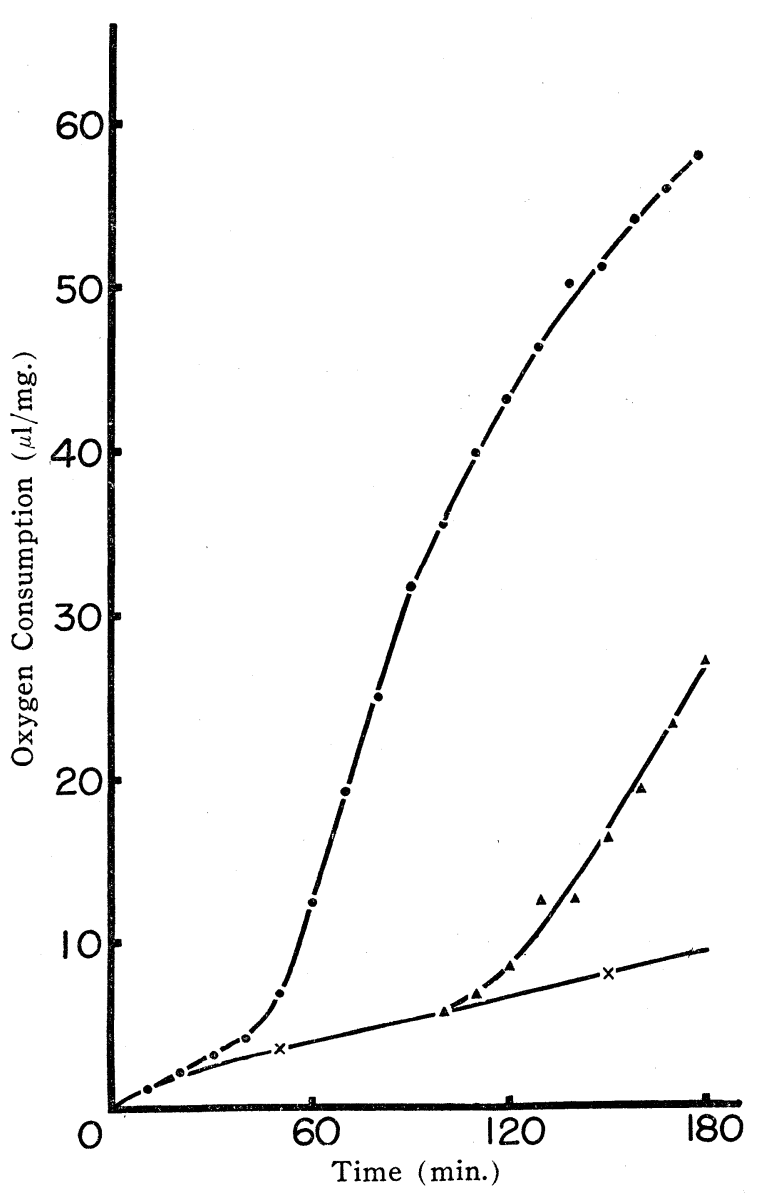

FIG. 4. Effect of 550 lux white light on the respiration of outersegments. All vessels contained $2.5 \mathrm{ml}$. of suspension of outersegments in Ringer solution without glucose, $p \mathrm{H}$ 7.4.

- $\bullet$, oxygen consumption in the dark.

$x-x$, oxygen consumption when exposed to 550 lux white light.

$\Delta-\Delta$, oxygen consumption of illuminated outersegments when placed in the dark after a 90-minute period. 


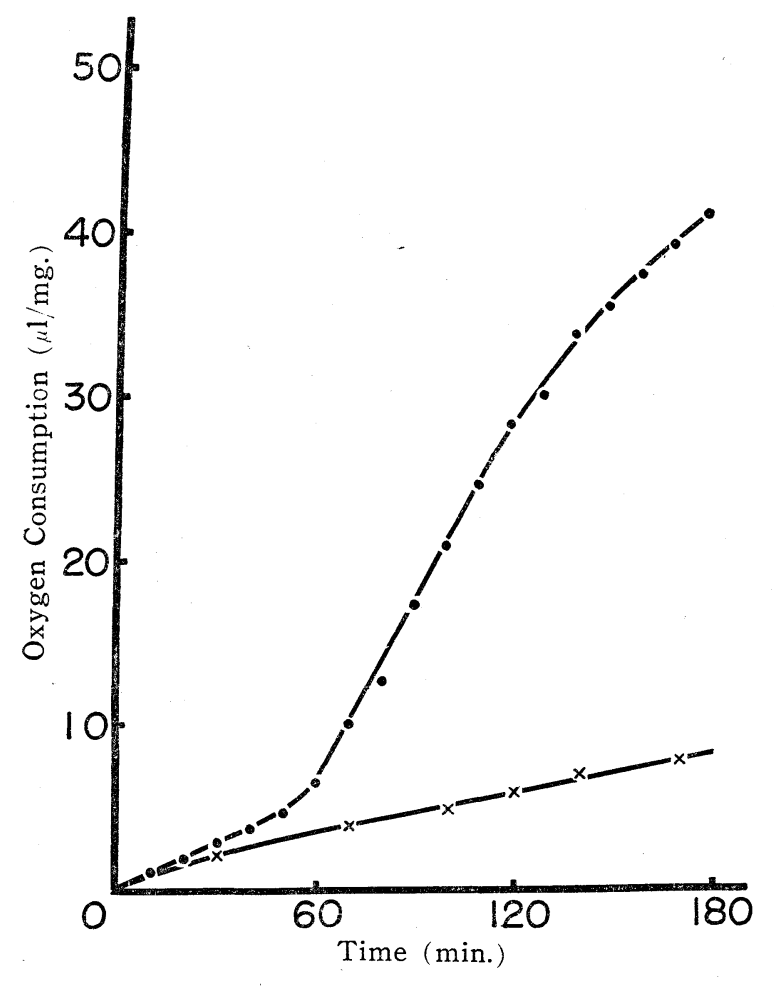

FIG. 5. Effect of 25 lux white light on the respiration of outersegments. All vessels contained $2.5 \mathrm{ml}$. of suspension of outersegments in Ringer solution without glucose, $p \mathrm{H} 7.4$.

- $\bullet$, oxygen eonsumption in dark condition.

$x-x$, oxygen consumption when exposed to 25 lux white light.

\section{3) Effects of illumination with bright white light before measurement}

The outersegment of rod contains a large amount of rhodopsin (chromoprotein), but it is bleached easily on exposure to light. Therefore, the outersegments containing bleached rhodopsin are prepared for the present measurement as follows:

(1) After a single cut is made right across the equator of the eye-ball, the hind half of it is placed in a vessel containing $0.65 \% \mathrm{NaCl}$ to be illuminated by bright white light, 650 lux during a 30-minute period. After the rhodopsin has been bleached completely, the outersegments are separated and collected by the floatation method described above. The oxygen consumption rate of this sample is shown in fig. 6. The significant phenomenon observed in this experiment is that oxygen consumption rates in the dark condition become lower than those of the measurement described above. But under illumination of 25 lux white light, the oxygen uptake of the same sample increases rapidly after the initial 60-minute period, and a remarkable inhibition as shown in fig. 5 is not observed. In the initial 60-minute period, the respiration of an illuminated sample (25 lux white light) is inhibited markedly in comparison with that of the dark one. But the inhibition is reduced gradually and after a 180-minute period, the respiration is inhibited about $21 \%$.

(2) The suspension of outersegments containing rhodopsin is divided equally into two parts and poured into experimental flasks separately. One part is kept 

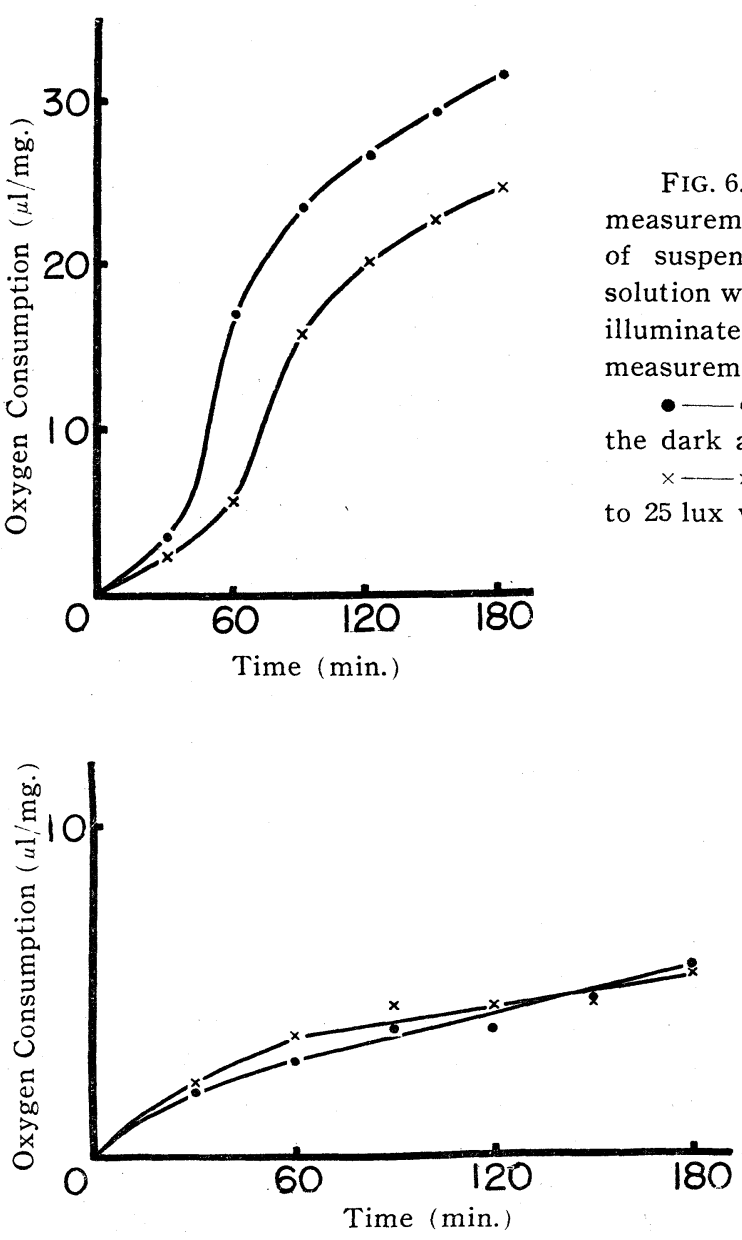

FIG. 6. Effect of bright white light before measurement. All vessels contained $2.5 \mathrm{ml}$. of suspension of outersegments in Ringer solution without glucose, $p \mathrm{H}$ 7.4. Samples were illuminated by white light, 650 lux before measurement.

- - , oxygen consumption when kept in the dark after the illumination.

$x-x$, oxygen consumption when exposed to 25 lux white light during the measurement.

FIG. 7. Effect of bright white light before measurement. All vessels contained $2.5 \mathrm{ml}$. of suspension of outersegments in Ringer solution without glucose, $p H$ 7.4. Oxygen consumption rates were measured under illumination of 550 lux white light.

- - oxygen consumption when kept in the dark before measurement.

$x-x$, oxygen consumption when exposed to flash of $10^{1} \mathrm{lu}$ men per sec. before measurement.

in the dark, the other exposed to flash of $10^{4}$ lumen per sec. at a distance of $10 \mathrm{~cm}$. and oxygen consumption rates of both samples are measured under illumination of 550 lux white light (fig. 7). No significant change between both results can be observed.

\section{4) Effects of temperature before measurement}

The suspension of outersegments is placed in two flasks, one of which is immersed in ice water $\left(2^{\circ} \mathrm{C}\right.$. $)$, and the other in warm water $\left(30^{\circ} \mathrm{C}\right.$. $)$ during a 15-minute period in the dark. The oxygen consumption rates of both samples are measured under illumination of 550 lux white light (fig. 8). It is demonstrated in fig. 8 that the oxygen uptake of outersegments immersed in warm water before measurement is inhibited during the first 90 -minute period, but thereafter the inhibition ceases and oxygen consumption increases rapidly. 


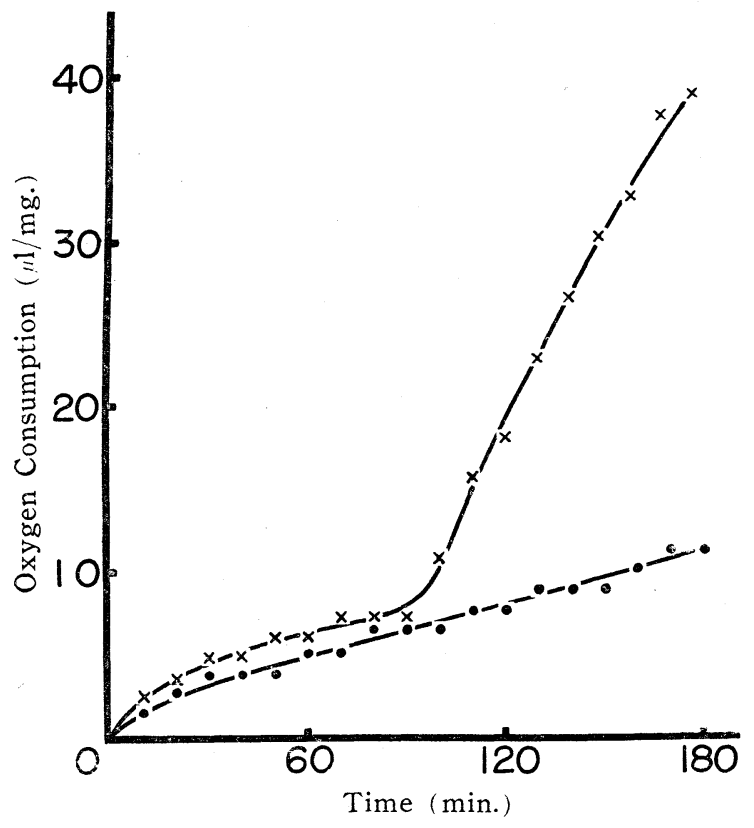

FIG. 8. Effect of temperature before measurement. The suspension of outersegments was placed in two flasks, the oxygen consumption rates of both samples were measured under illumination of 550 lux white light.

- - oxygen consumption when immersed in ice water $\left(2^{\lrcorner}\right.$C. ) during a 15 minute period.

$x-x$, oxygen consumption when immersed in warm water $\left(30^{\circ} \mathrm{C}\right.$. ) during a 15 -minute period.

\section{5) Effects of some metabolic inhibitors}

Pottasium cyanide $\left(2 \times 10^{-3} \mathrm{M}\right)$, sodium fluoride $\left(2 \times 10^{-3} \mathrm{M}\right)$ and sodium iodoacetate $\left(5 \times 10^{-3} \mathrm{M}\right)$ are used in the experiments. In each experiment, one flask containing $2 \mathrm{ml}$. suspension of outersegments in a main vessel and $0.5 \mathrm{ml}$. of Ringer solution containing an inhibitor in the side arm, and another flask is used as a control containing Ringer solution alone in the side arm. The solution in the side arm is tipped in after $10 \mathrm{~min}$. equilibration.

The respiration of outersegments is sensitive to potassium cyanide both in the dark and illuminated conditions (fig. 9). Both flasks are illuminated by 550 lux white light during the first 60-minute period, then they are kept in the dark during the last 60 -minute period. In the light period, respiration is inhibited

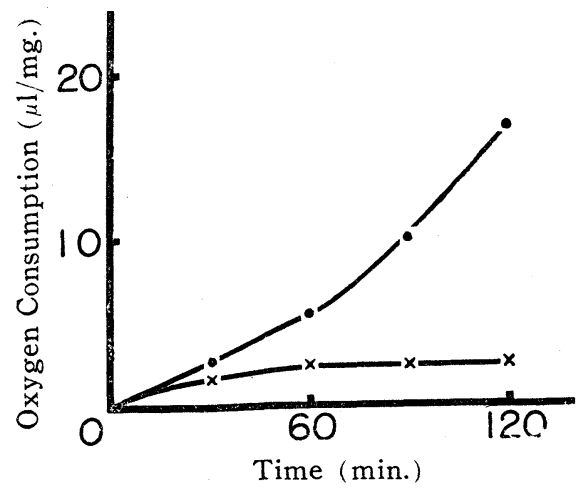

FIG. 9. Effect of potassium cyanide on the respiration of outersegments. One vessel (- ) contained $2.5 \mathrm{ml}$. of suspension of outersegments in Ringer solution without glucose, $p \mathrm{H} 7.4$, the other $(x-\times)$ contained $2.0 \mathrm{ml}$. of suspension of outersegments in Ringer solution without glucose and $0.5 \mathrm{ml}$. of $10^{-2} \mathrm{M}$ potassium cyanide Ringer solution. Measurement was carried out being exposed to 550 lux white light during the first 60 minute period and then in the dark condition. 
$59 \%$ by $2 \times 10^{-3} \mathrm{M}$ potassium cyanide, and in the dark period, inhibited $100 \%$. $2 \times 10^{-3} \mathrm{M}$ sodium fluoride showes no noticeable effect on oxygen consumption rates in the first 90-minute period under illumination by 550 lux white light, but as soon as this sample is kept in the dark after measurement of the first 90-minute period, its oxygen consumption rate is increased rapidly (fig. 10 ).
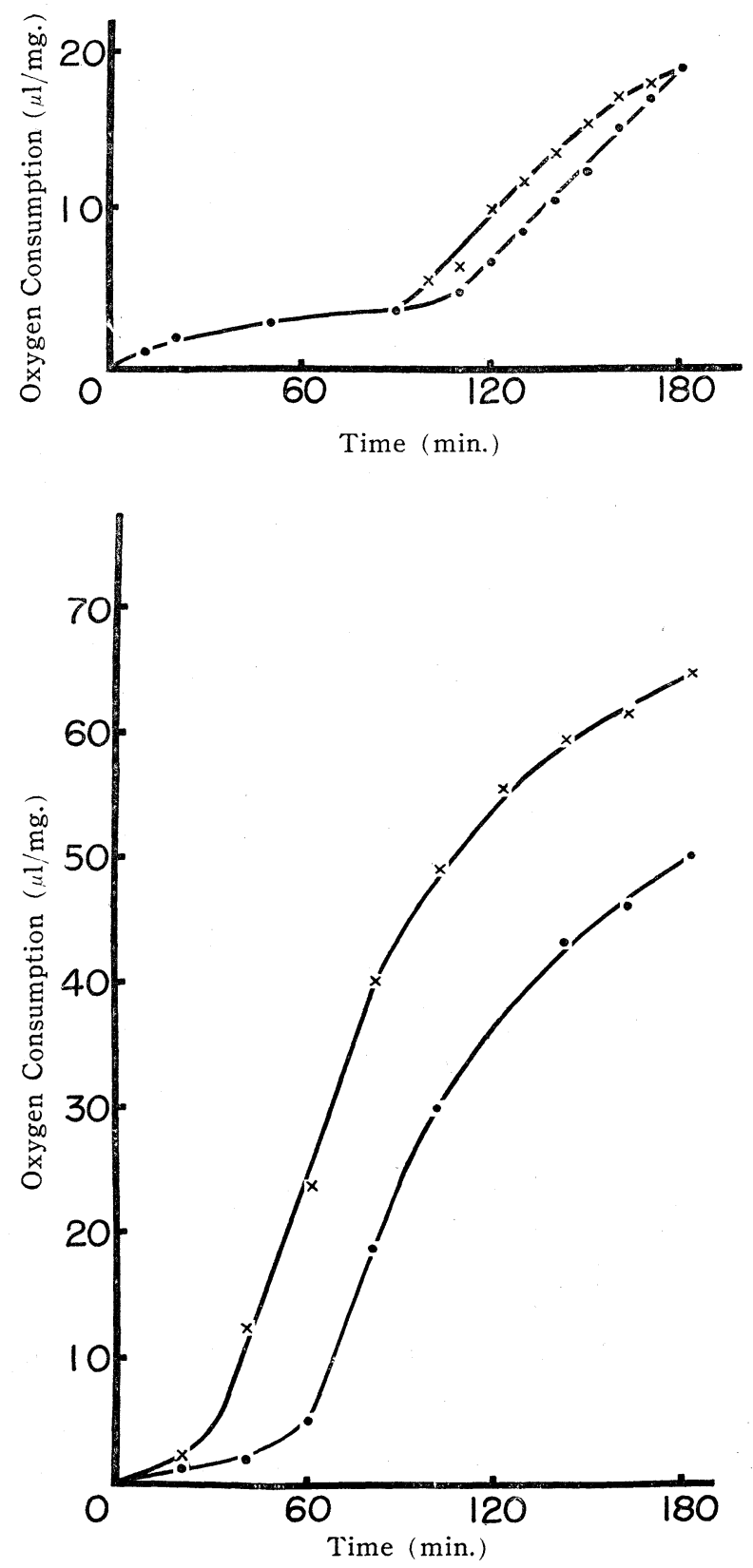

FIG. 10. Effect of sodium fluoride on the respiration of outersegments. One vessel (- ) contained $2.5 \mathrm{ml}$. of suspension of outersegments in Ringer solution without glucose, $p \mathrm{H} 7.4$, the other $(x-x)$ contained $2.0 \mathrm{ml}$. of suspension of outersegments in Ringer solution without glucose and 0.5 $\mathrm{ml}$. of $10^{-2} \mathrm{M}$ sodium fluoride Ringer solution. Measurement was carried out being exposed to 550 lux white light during the first 90-minute period and then in the dark condition.

FIG. 11. Effect of sodium iodoacetate on the respiration of outersegments in the dark condition. One vessel (- $\bullet$ ) contained $2.5 \mathrm{ml}$. of suspension of outersegments in Ringer solution

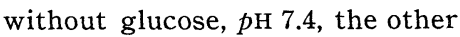
$(x-x)$ contained $2.0 \mathrm{ml}$. of suspension of outersegments in Ringer solution without glucose and $0.5 \mathrm{ml}$. of $2.5 \times 10^{-2} \mathrm{M}$ sodium iodoacetate Ringer solution. 


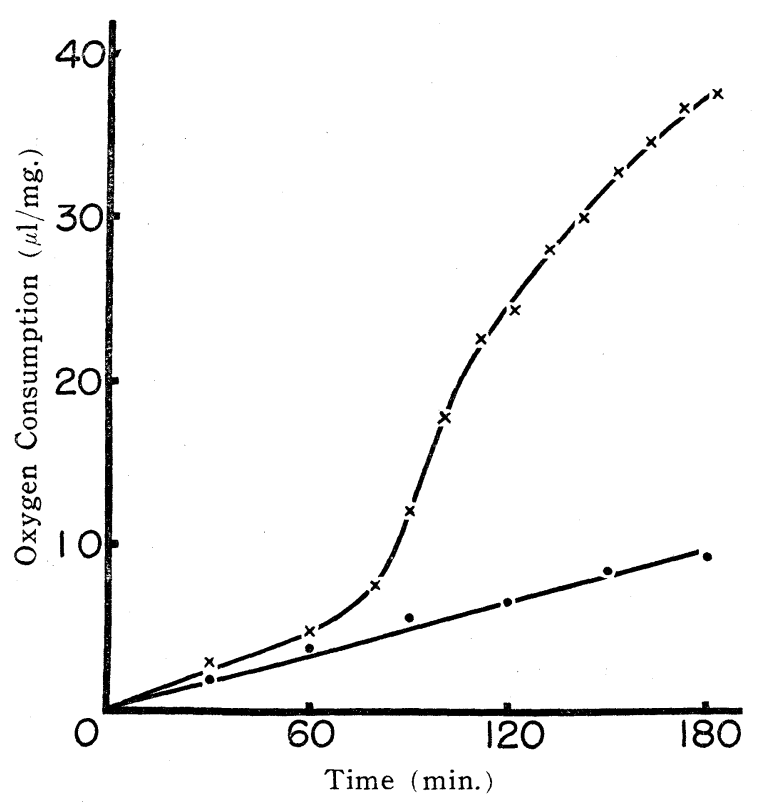

FIG. 12. Effect of sodium iodoacetate on the respiration of outersegments in the illuminating condition (550 lux white light). One vessel (- $\bullet$ ) contained $2.5 \mathrm{ml}$. of suspension of outersegments in Ringer solution without

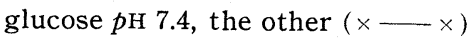
contained $2.0 \mathrm{ml}$. of suspension of outersegments in Ringer solution without glucose and $0.5 \mathrm{ml}$. of 2.5 $\times 10^{-2} \mathrm{M}$ sodium iodoacetate $\mathrm{Rin}$ ger solution.

The effect of $5 \times 10^{-3} \mathrm{M}$ sodium iodoacetate is shown in fig. 11 and 12 . In the dark condition, iodoacetate eliminates the inhibition of oxygen uptake in the early stage about $20 \mathrm{~min}$. earlier than the control and increases the oxygen consumption rate $30 \%$ after a 180 -minute period (fig. 11). In the light condition (550 lux white light), iodoacetate showes a slight increase in the respiration of outersegment during the first 70 -minute period, but then the inhibition due to illumination ceases and the oxygen consumption rate increases rapidly during the last 110-minute period (fig. 12).

\section{DISCUSSION}

There are numerous reports on the respiration of whole retina. But any common conclusion does not seem to have been accepted yet as to effects of illumination upon oxygen consumption rates. A number of authors observed that the respiration of the vertebrate retina is slightly inhibited by illumination, while some denied the influence of illumination (11). The cause of the diversity in their experimental results remains unexplained. In our experiments the respiration of outersegments of rods reveals a remarkable difference between dark and light conditions. The dry weight of separated outersegments of rods is proved about $1 / 50$ to $1 / 100$ that of the whole retina in our experiment. Therefore, in the study using whole retina, the proper response of outersegments themselves may have been overlooked.

As shown in fig. 3, dark adapted outersegments reveal a slight increase in oxygen consumption at the early stage under 1 lux white light when compared with that of the dark one. The rhodopsin is bleached slowly by such dim light, 
so it is presumed that the metabolism of outersegments is influenced by rhodopsin during its bleaching.

Twenty-five or 550 lux white light inhibits strongly the oxygen consumption of the outersegment (fig. 4 and 5). In these conditions rhodopsin is rapidly bleached. From these results, it may be postulated that another photochemical reaction occures in the outersegment during illumination without rhodopsin. The same observation is shown in fig. 7. Svaetichin (18) suggested that the rods are not out of function in strictly photopic conditions, but they seem to play an important role in pursuing in day light vision. This suggestion may be a clue to interpret a physiological meaning of our experimental results. Hosoya (6) asertained that the regeneration of frog rhodopsin in vivo is retarded in a low atomospheric pressure, but if the oxygen is supplied sufficiently, the rate of regeneration is normal. The same observation was reported by Zewi (20). However, the bleached rhodopsin can not be regenerated in the suspension of outersegments. Wald and Hubbard (19) suggested that the apoenzyme, called retinene reductase, may be contained in the outersegment of the rod. They observed that the reduction of retinene is performed in the isolated retina or in vitro irreversily, with Co I.

Kühne (4), Garten (5), Andogsky (1), Hosoya and Sasaki (7) reported that some special substance in the pigmented epithelium of retina is necessary for the regeneration of rhodopsin, but Hwang (9) could not demonstrate any significant difference of the respiration of the frog retina between the experiments with or without the pigmented epithelium. From these results and our observations, a large oxygen consumption of the outersegment in the dark condition does not seem due to the regeneration of rhodopsin and the oxidation of vitamin A into retinene, but due to some water-soluble metabolite contained in outersegments.

Recently, Hubbard (8) measured the respiration of isolated outersegments of rods of the frog retina by the Cartesian diver method. She concluded that $Q \mathrm{O}_{2}$ of the outersegment is about $-0.7 \mu \mathrm{l}$. per $\mathrm{mg}$. dry weight per hour at $20^{\circ} \mathrm{C}$. and the respiratory rate is independent of the state of dark adaptaion of the outersegment. This value is smaller than the $Q_{\mathrm{O}}$ of outersegments illuminated during measurements in our experiment. The diversity between these results may be due to the following facts: (1) difference in the separating method, (2) temperature and required time during operation, (3) temperature during measurement.

Recently, Arden (2) reported that a hypertonic sucrose solution is unable to extract water from the outersegment. Therefore, the loss of metabolites of the outersegment separated by our sugar floatation method may be negligible at low temperatures. On the contrary, Hubbard (8) collected outersegments by gently scraping the posterior surface of the retina with a spature in Ringer solution, so it took about 2 or 3 hours in the dark room. Although the temperature during the operation of her experiment is unknown, water-soluble metabolite may escape into surrounding solution during such a long time. For this reason, the low $\mathrm{QO}_{2}$ value might be observed during the dark adaptation.

Each respiration in dark and light conditions is sensitive to potassium cya- 
nide. This result corresponds with that of Hubbard (8).

On an electrophysiological study, Noell (12) observed that in winter frogs, when decapitated 5 to 20 minutes after injection of $0.5 \mathrm{mg}$. iodo acetate $/ 100 \mathrm{~g}$. B.W. or more, an electroretinogram disappeared rapidly within one to two minutes at $21^{\circ} \mathrm{C}$. And he found histologically (13) that rod, cone and pigment cells are affected irreversibly by intravenous administration of iodoacetate. Therefore, it is supposed that iodoacetate has an immediate effect on the metabolism of the outersegment of the rod related to visual functions.

According to Barron (3), phosphoglyceraldehyde dehydrogenase and alcohol dehydrogenase are inhibited $90 \%$ and succinic dehydrogenase is inhibited $42 \%$ by $5 \times 10^{-3} \mathrm{M}$ iodoacetate. It is unknown whether a glycolitic cycle is present in the outersegment of the rod, but the rate of respiration of the light-adapted outersegment is increased by the addition of $0.01 \mathrm{~m}$ fructose diphosphate (8).

The isolated rod outersegment suspended in buffer solution can not convert retinene to vitamin $\mathrm{A}$, but in the presence of a crude preparation of fructose diphosphate they are enabled to perform a partial conversion (19). Wald and Hubbard (19) suggested that the outersegment may be able to degrade fructose-diphosphate fermentatively. However, if this effect of iodoacetate on outersegments were attributable to the inhibition of glycolysis, especially phospho-glyceraldehyde dehydrogenase, the oxygen consumption of them treated with iodoacetate would not increase as shown in fig. 11. From the view point of respiration of outersegments, iodoacetate seems to function to remove the inhibition caused by illumination. Therefore, iodoacetate may have an effect on the photochemical reaction which is unassociated with rhodopsin in the outersegment.

\section{SUMMARY}

(1) A study on the respiration of the isolated outersegment of rod separated from toad's retina was made by means of the Warburg apparatus.

(2) In the dark condition, the $Q_{\mathrm{O}}$ of isolated outersegment reveals a high value about $-30 \mu 1$. at $26^{\circ} \mathrm{C}$.

(3) The oxygen consumption rate is inhibited remarkably following illumination ( 25 or 550 lux white light).

(4) Effects of bright white light and temperature before the measurement have been examined.

(5) Effects of some inhibitors on the respiration of the outersegment have been discussed.

The expenses for this study was defrayed by a grant from the Ministry of Education.

\section{REFERENCES}

1. Andogsky, N. v. Graefes Arch. f. Ophthalm. 44: 404, 1897.

2. ARden, G. B. J. Physiol. 123 : 377, 1954.

3. BARron, E. S. G. Advances in Enzymology, Vol. XI, p. 201. New York: Interscience, 1951.

4. EWALd, A. AND KüHne, W. Unters. Physiol. Inst. Heidelb. 1 : 139, 248, 370, 1877-8. 
5. Garten, S. v. Graefes Arch. f. Ophthalm. $63:$ 112, 1906.

6. Hosoy A, Y. unpublished data.

7. Hosoy A, Y. AND SASAKI, T. Tohoku J. exp. Med. 32: 447, 1938.

8. Hubbard, R. J. gen. Physiol. $20: 373,1954$.

9. Hwang, T. Jap. J. Physiol. 1 : 169, 1950.

10. Kimura, E. Jap. J. Physiol. 3 : 25, 1952.

11. NakAshima, M. Momaku no Kagaku (Chemistry of the Retina). Tokyo: Sogensha, 1948.

12. Noell, W. K. J. cell. comp. Physiol. $37: 283,1951$.

13. NOELL, W. K. ibid. $40: 25,1952$.

14. Ottoson, D. And Svaetichin, G. Acta Physiol. Scand. 29 : 31, 1953.

15. Ottoson, D. AND Svaetichin, G. ibid. 29 : supp. 106, 583, 1953.

16. SJÖstrand, F. S. J. cell. comp. Physiol. $42: 15,1953$.

17. SJÖSTRAND, F. S. ibid. $42: 45,1953$.

18. Svaetichin, G. Acta Physiol. Scand. 29 : supp. 106, 601, 1953.

19. Wald, G. AND Hubbard, R. J. gen. Physiol. 32: 367, 1949.

20. ZEWI, M. Acta Soc. Sci. Finn. N.S.B. 2: No. 4, 1939. 